\title{
EL PROBLEMA DE ALHACÉN
}

\section{Carlos Alberto Cardona Suárez*}

\author{
Universidad del Rosario (Colombia)
}

\section{RESUMEN}

El artículo se ocupa del problema que Alhacén formuló y resolvió en el libro V de su tratado de óptica. A saber, encontrar el punto de reflexión en un espejo dado, si se conocen las posiciones del objeto y del observador. Se pretende, primero, exponer el complejo esquema de argumentación usado por el filósofo y científico árabe, y segundo, proponer un posible esquema de razonamiento como un candidato que exhibe la lógica del descubrimiento.

PALABRAS CLAVE: Problema de Alhacén. Espejo esférico convexo. Reflexión. Percepción. Pirámide visual.

\section{ALHACEN'S PROBLEM}

\section{ABSTRACT}

This paper deals with the problem that Alhazen formulated and solved in book V of his treatise on optics. That is to say, finding the reflection point in a given mirror, when the positions of both the object and the observer are known. The intention is to, firstly, exhibit the complex argumentation scheme used by the Arabic scientist and philosopher; and secondly, propose a possible method of reasoning like a candidate exhibiting the logic of the theory which could lead to the discovery of the solution.

KEY WORDS: Alhazen's problem. Convex spherical mirror. Reflection. Perception. Visual pyramid.

A finales del siglo XII o comienzos del XIII un fantasma inició su recorrido por Europa. Nos referimos a la traducción del árabe al latín de un extenso y profundo tratado de óptica. Los pasos de dicho fantasma se sienten palpitar en las obras de Roger Bacon (1214-1294), John Pecham (1230-1292) y Erazmus Ciolek Witelo (1230-1314), para nombrar las más importantes. No hay rasgos del personaje que emprendió la tarea de la traducción y las fuentes ya parecen desestimar que se tratara de Gerard de Cremona. ${ }^{1}$ Nos referimos,

* Profesor Titular, Escuela de Ciencias Humanas, Universidad del Rosario, Bogotá, Colombia, doctor en filosofía. Dirección para correspondencia: Carrera 68 A, No. 22 A 75, casa 
pues, al tratado cuyo título en árabe es Kitāb al-Manāzir y que se atribuye a Ibn al-Haytham. Se han propuesto dos títulos para la presentación de la obra en latín De «Aspectibus» y «Perspectiva». El primero se puede traducir como «Acerca de las apariencias» para darle realce a los aspectos psicológicos que menciona el tratado. En tanto que el segundo se puede traducir como «Óptica» subrayando la concentración en los medios por los cuales la visión se lleva a cabo. El nombre completo del autor es Abu 'Ali al-Hasan ibn al-Hasan ibn al-Haytham, nombre que se ha abreviado bajo una de dos formas Alhacén o Alhazen. Después del año 1250 varias copias manuscritas del De aspectibus llegaron a instalarse en puntos estratégicos de Europa. La primera edición impresa apareció en 1572 con la excelente obra de Friedrich Risner Opticae thesaurus. Ésta edición permitió contar en Europa con una versión canónica del trabajo de Alhacén; versión que se constituyó en una de las fuentes de inspiración de Kepler, Descartes y Huygens entre otros. No obstante, la versión latina tiene algunas deficiencias. Los tres primeros capítulos del libro I en la versión original en árabe, allí donde se establecen algunos criterios metodológicos, no están presentes en la versión europea. Lagunas similares se encuentran en los libros restantes. En consecuencia, la versión en latín no es una réplica exacta de la versión árabe. Los tres primeros libros de la versión original en árabe han sido traducidos al inglés recientemente por A. I.Sabra; en tanto que la versión latina ha sido llevada al inglés por Mark Smith. ${ }^{2}$ Para

7, Bogotá (Colombia). Correo electrónico: carlos.cardona@urosario.edu.co. Agradezco profundamente los comentarios y contribuciones de Sebastian Cristancho y Nicolás Montenegro. La presente investigación contó con el apoyo del Fondo de Investigaciones de la Universidad del Rosario FIUR (código del proyecto: DVG-073). Las figuras se han construido con los programas Cabri II-plus y Cabri 3D.

1 SMith, M. (2001), Alhacen's Theory of visual perception, traducción al inglés con comentarios de los tres primeros libros del De Aspectibus, 2 vols., Philadelphia, American Philosophical Society, p. xx. Hay indicios de una traducción del De Aspectibus al italiano en el siglo XIV. El escultor italiano Lorenzo Ghiberti reportó haber tenido contacto con las ideas de Alhacén (Cfr. StefFens, B. (2007), Ibn al-Haytham First Scientist. Greensboro, Morgan Reynolds, p. 104). La primera alusión a una versión en latín en Occidente proviene de un escrito de Jordanus de Nemore en un período entre 1220 y 1230; cfr. SABRA, A. I., (1982), Ibn al-Haytham's lemmas for Solving 'Alhazen's Problem', Archive for History of Exact Sciences, 26, (4), 299-324, p. 299.

2 Smith, M. (2001), Alhacen's Theory of visual perception, traducción al inglés con comentarios de los tres primeros libros del De Aspectibus, 2 vols., Philadelphia, American Philosophical Society. SMITH, M., (2006), Alhacen on the principles of reflection, traducción al inglés con comentarios de los libros 4 y 5 del De Aspectibus, 2 vol., Philadelphia, American Philosophical Society. SMith, M., (2008), Alhacen on image-formation and distortion in 
los efectos asociados con el tema del presente artículo, tomaremos en consideración la traducción al inglés que de la versión en latín preparó Mark Smith. ${ }^{3}$

Alhacén nació muy probablemente en el año 965 en Basora (Al-Basra), ciudad localizada en lo que hoy se conoce como Irak. Basora es también el puerto de Simbad el Marino. Alhacén participó como estudiante en la Casa de la Sabiduría, una de las más grandes bibliotecas del mundo musulmán, fundada en el siglo IX con el objeto de promover el estudio y traducción de obras clásicas. Allí tuvo la oportunidad de familiarizarse con las obras de Platón, Aristóteles, Euclides, Apolonio, Ptolomeo y Galeno. Alhacén llegó a ocupar un cargo público en Basora; cargo que abandonó aduciendo, según algunos comentaristas, razones asociadas con algún tipo de enfermedad mental. ${ }^{4} \mathrm{El}$ científico árabe se trasladó después (1010) al Cairo para trabajar bajo el gobierno de al-Hakim, quien ordenó la construcción de la biblioteca del Cairo. Todo parece indicar que la relación estaba fundada en un plan novedoso que Alhacén había concebido para controlar las devastadoras crecientes del Nilo. Ciertos desacuerdos con al-Hakim, posiblemente asociados con el fracaso del proyecto para controlar el Nilo, fueron tejiendo las condiciones para que Alhacén fuese condenado a arresto por cerca de diez años. Es probable que durante ese tiempo se hubiese concebido y adelantado buena parte del proyecto de su tratado de óptica. Una vez terminó el arresto, Alhacén se instalo en El Cairo para posteriormente desplazarse a Bagdad y Basora. El filósofo y científico árabe murió en el año $1040 .^{5}$

El De Aspectibus es un compendio de 7 libros. El libro I presenta un esbozo general de la teoría de la visión de Alhacén. El libro II da cuenta de su forma peculiar de concebir la psicología de la percepción. Este libro sienta las bases teóricas para que el libro III se ocupe de los errores inducidos en la percepción visual cuando está en juego la visión directa. Los libros IV y V se ocupan de la reflexión y formación de imágenes en espejos planos y en espejos curvos. En estos libros se enuncia y se resuelve el famoso Problema de

mirrors, traducción al inglés con comentarios del libro 6 del De Aspectibus, 2 vol., Philadelphia, American Philosophical Society. SMITH, M. (2010), Alhacen on refraction, traducción al inglés con comentarios del libro 7 del De Aspectibus, 2 vol., Philadelphia, American Philosophical Society.

3 Las referencias a la obra de Alhacén aludirán al libro en números romanos, seguido del capítulo en números arábigos $\mathrm{y}$, después del punto, la numeración del párrafo correspondiente.

4 StefFens, B. (2007), p. 44.

5 SABRA, A. I., (2003), Ibn al-Haytham, brief life of an Arab Mathematician: died circa 1040, en http://www.harvardmagazine.com/on-line/090351.html. Algunos comentaristas tienen dudas acerca del regreso del filósofo a su patria natal, LiNDBERG, D. C., (1967), Alhazen's Theory of Vision and its Reception in the West, Isis, 58, (3), pp. 321-341, p. 60. 
Alhacén. El libro VI - complemento del III- se ocupa de los errores en la percepción visual ocasionados por rayos reflejados. Por último, el libro VII se detiene en el estudio de la refracción de la luz.

No es difícil establecer con claridad las principales influencias en la obra de Alhacén. Aristóteles contribuye para establecer una actitud y, quizá, un método para la investigación científica en general. Euclides y Apolonio aportan el trasfondo geométrico. La obra de Ptolomeo sugiere problemas y aporta la dirección específica en la que han de enfrentarse. En muchos casos, Alhacén se limita a servir de correa de transmisión de las ideas de Ptolomeo. Por último, la anatomía del ojo se toma casi directamente de los trabajos de Galeno. En el contexto árabe, Ya 'qūb al-Kindi (801-866) contribuyó a la asimilación del pensamiento griego e inició la osadía de participar en dicha empresa con una mirada crítica. Si bien Aristóteles se había sentido inclinado a pensar que el corazón podía ser el asiento del alma, Galeno se atrevió a sostener que las funciones más importantes asociadas al alma debían tener su asiento en el cerebro. Los pensadores árabes quisieron ofrecer una descripción galenizada de la psicología de Aristóteles. Ellos quisieron asignar ciertas facultades psicológicas a regiones específicas del cerebro. Una buena parte de esta síntesis, estudiada por Alhacén, se encuentra en Los diez tratados del ojo escrito por Hunayn ibn Ishāq (809-873).

Sin el ánimo de ser exhaustivos, podemos reducir a seis los aportes básicos de Alhacén en el De Aspectibus: (i) ofrecer argumentos definitivos en favor del intramisionismo; (ii) elucidar la naturaleza compleja del vértice en la pirámide visual euclidiana; (iii) aportar una descripción psicológica muy completa de los procesos de inferencia presentes en la percepción directa; (iv) advertir la importancia de métodos experimentales y modelos matemáticos para validar conjeturas en el estudio de la óptica; (v) proponer y ofrecer una solución completa del Problema de Alhacén; (vi) reiniciar los estudios cuidadosos de la refracción.

(i) Ofrecer argumentos definitivos en favor del intramisionismo. A propósito de los fenómenos asociados con la percepción visual, los griegos se debatían entre, por un lado, concepciones extramisionistas (Platón, Euclides, Ptolomeo) que asumen que un cierto efluvio que emana del ojo sale al encuentro del objeto hasta entrar en contacto directo con él, provocando después la aparición de una imagen o fantasma del objeto contemplado; $\mathrm{y}$, por otro lado, concepciones intramisionistas (Aristóteles, atomistas) que abogan porque alguna modificación del objeto sobre el medio sea la responsable de que el ojo reciba la forma sensible del objeto correspondiente. Alhacén, por su parte, ofreció argumentos poderosos en favor del intramisionismo y mostró cómo 
podía usarse en este caso el modelo de la pirámide euclidiana, concebida inicialmente para esquemas extramisionistas. En efecto, si pensamos que es a partir del ojo que emana cierto efluvio visual, conviene preguntar si hay algo que regresa al ojo o nada retorna. En el segundo caso nada podría percibirse. En el primero nos vemos obligados a restituir la tesis intramisionista. También conviene preguntar si ese efluvio es o no corporal. Si es corporal, hemos de admitir algo absurdo; esto es que una sustancia corporal que emana del ojo puede llenar en un solo momento todo el espacio que tenemos al frente desde nuestros ojos hasta la inmensidad del cielo, sin que el ojo sienta mengua alguna en su constitución. Si no es corporal no hay espacio para hablar de sensación, toda vez que ella implica el reconocimiento de objetos físicos por la afección que ellos producen en nuestros órganos corporales (Alhacén, I, 6.56).

(ii) Elucidar la naturaleza compleja del vértice en la pirámide visual euclidiana. La pirámide visual de Euclides es un instrumento que, sin duda, simplifica el análisis de la visión. En el caso de Euclides lo fundamental es: (i) un observador reducido a un punto (el vértice de la pirámide); (ii) un objeto que ha de ser contemplado y que constituye la base de la pirámide; (iii) una mediación que ocurre en virtud de líneas rectas entre el objeto y el observador; y (iv) un observador que lee las claves geométricas de la mediación para inferir posiciones, tamaños y distancias del objeto percibido. Ahora bien, concebir el observador como un punto geométrico es una simplificación que deja por fuera aspectos esenciales. Un punto, según Euclides, es aquello que no tiene partes. Concebir el ojo como un algo sin partes no nos permite abrazar la complejidad que en sí encierra la percepción. Alhacén, considerando la información anatómica aportada por Galeno, procuró establecer la funcionalidad geométrica de cada una de las partes del ojo.

(iii) Aportar una descripción psicológica muy completa de los procesos de inferencia presentes en la percepción directa. El ojo es una ventana abierta que permite instalar en la pared posterior del cristalino una imagen isomórfica del objeto contemplado. A continuación esta imagen es capturada, sentida y transportada por los espiritus visuales a través del nervio óptico hasta el nervio común y por éste hasta la parte frontal del cerebro sin pérdida del isomorfismo inicial. Allí el sensor central está en condiciones de percibir las propiedades visibles, algunas de ellas encarnadas - en el buen sentido aristotélicoen los objetos exteriores. Entre estas propiedades conviene asentar la siguiente distinción. De un lado se encuentran las propiedades que se perciben por la sensación bruta [sensu solo] y, de otro lado, las propiedades que son percibidas gracias a la intervención del juicio, del reconocimiento y de la diferencia- 
ción en conjunción con la sensación de las formas percibidas. Alhacén adelantó un juicioso estudio de las características de las inferencias presentes en la percepción visual. ${ }^{6}$

(iv) Advertir la importancia de métodos experimentales y modelos matemáticos para validar conjeturas en el estudio de la óptica. Todas las superficies reflectantes desvían la luz, forma y color. Lo hacen ajustadas a dos principios centrales: (i) dado un punto de reflexión sobre la superficie ocurre que la línea de incidencia y la línea de reflexión se encuentran en el mismo plano con la normal trazada a la superficie en el punto de reflexión; y (ii) tales líneas forman con la normal ángulos equivalentes (Alhacén, IV, 3.2). El filósofo árabe no se limitó a sugerir plausibles argumentos especulativos o a apoyarse en la tradición (Aristóteles, Euclides, Ptolomeo) para soportar las mencionadas leyes, sino que intentó someterlas a una rigurosa verificación empírica. Para ello dispuso de un cilindro macizo, sellado completamente, cuya base inferior contenía el dibujo de rectas de guía convergentes en el centro de la base del cilindro prefigurando el paso de la luz. Las paredes del cilindro poseían orificios justo en los puntos de intersección de las líneas de guía de la base y las paredes del cilindro. Estos agujeros se sellaban con bloques de madera fácilmente removibles. En la base se podía disponer un espejo en una posición controlada por el experimentador. Alhacén destapaba un agujero para permitir la entrada controlada de la luz y, después, retirando uno a uno cada tapón, buscaba la trayectoria de salida del rayo de luz reflejado.

De otra parte, Alhacén, siguiendo a Euclides y a Ptolomeo, se esmeró por hacer de la geometría el canon con el que debían presentarse los problemas y las soluciones asociadas con el estudio de los fenómenos ópticos. Así, la geometría logra encarnarse en el estudio de la percepción visual. No se trata de un instrumento adicional que pudiese hacernos la vida más cómoda. La geometría es el lenguaje en el que se puede formular significativamente las preguntas propias que atañen al estudio de la percepción visual.

(vi) Reiniciar los estudios cuidadosos de la refracción. Alhacén intentó darle continuidad a los estudios que Ptolomeo había iniciado a propósito de las regularidades matemáticas implícitas en los fenómenos de refracción de la luz. Él advirtió los problemas de Ptolomeo en el momento de pretender explicar el aumento del tamaño aparente de la Luna cuando era contemplada en el horizonte. Cuando un observador en la Tierra dirige su mirada al cielo, asimila la superficie

6 No carece de fundamento pensar en una anticipación a algunas claves fenomenológicas en la presentación de Alhacén. EL-BIZRI, N. (2005), A philosophical perspective on Alhazen's optics, Arabic Sciences and Philosophy, 15 pp. 189-218. 
celeste a un plano que se extiende indefinidamente en todas las direcciones. Así las cosas, cuando contempla la Luna en el horizonte, la mente habrá grabado la expectativa que impone que la distancia a la Luna es bastante mayor comparada con la distancia recordada en el zenit. Si en los dos casos imaginamos objetos que poseen el mismo tamaño absoluto, ellos serán contemplados bajo conos visuales de la misma amplitud angular; en consecuencia, como en el horizonte la Luna parece más distante, la facultad visual aventurará la hipótesis según la cual, la Luna debe ser un objeto de mayor amplitud en su tamaño.

\section{EL PROBLEMA DE ALHACÉN}

Después de ofrecer argumentos y evidencias experimentales en favor de las leyes de la reflexión, y después de fortalecer la confianza en el principio de Ptolomeo, ${ }^{7}$ Alhacén propone el famoso problema que lleva su nombre. Este problema reza así: si yo conozco la geometría del espejo y las ubicaciones del objeto y del observador, se pide hallar el punto de reflexión, en caso de existir, para que después se pueda establecer la ubicación precisa de la imagen correspondiente. ${ }^{8}$ Este problema se formuló en forma precisa inicialmente en la proposición 18 del libro V.$^{9} \mathrm{Si}$ el espejo es plano, la solución es trivial. En los casos restantes, la solución exige una complejidad que no alcanza a advertirse en la simplicidad de la formulación del problema. ${ }^{10}$ Nos

7 Este principio, formulado en lenguaje intramisionista, asegura que la imagen que de un objeto contempla un observador en una posición conocida frente a un espejo, debe hallarse en la intersección de la prolongación del rayo visual que llega al observador y de la perpendicular trazada desde el objeto al plano tangente a la superficie del espejo en el punto de incidencia; cfr. Smith, M., (1996), Ptolemy's Theory of Visual Perception, traducción al inglés de la Óptica, Philadelphia, The American Philosophical Society, III, §3. Alhacén V, 2.1.

8 Los autores más representativos de la geometría griega mantienen una distinción entre problema y teorema. Un problema se presenta gramaticalmente en infinitivo y pide la construcción de un objeto geométrico en un contexto claramente delimitado. Un teorema, por su parte, se presenta bajo la forma de un condicional que pretende aseverar una propiedad en una configuración geométrica dada. Cfr. KNORR, W. R., (1986), The Ancient Tradition of Geometric Problems, New York, Dover Publications, INC, p. 348.

9 Alhacén V, 2.137.

10 En la presentación que Marcus Baker hace de la bibliografía acerca del tema, califica así la propuesta de Alhacén: «La solución se lleva a cabo con la ayuda de una hipérbola que interseca una circunferencia, y resulta excesivamente prolija e intrincada» (BAKER, M., (1881), Alhazen's Problem. Its Bibliography and an Extension of the Problem, American Journal of Mathematics, Vol. 4, (1), pp. 327-331, p. 327. 
concentraremos en la solución para el caso de los espejos convexos (esféricos, cónicos y cilíndricos).

Alhacén divide el problema en dos casos. El primero de ellos es muy sencillo; mientras que el segundo reviste una complejidad extrema. En el primero caso se establece como condición que tanto objeto como observador se encuentren a la misma distancia del centro del espejo. Imaginemos que $O$ representa la posición del objeto y $V$ la del observador frente a un espejo esférico convexo de centro en $N$ (Figura 1). Imaginemos también que $O N \cong V N$. La circunferencia $A G B$ define el corte de la esfera con el único plano que contiene a los puntos $O, V$ y $N$. Los puntos $A$ y $B$ definen los límites del sector efectivo para la reflexión. ${ }^{11}$ Se traza la bisectriz $N G M$ al ángulo $O N V$. Esta bisectriz se encuentra en el plano $O N V$ y corta ortogonalmente la circunferencia en $G$. Los triángulos $O G N$ y $V G N$ son obviamente congruentes y, en consecuencia, los ángulos $O G M$ y $M G V$ resultan congruentes; esto convierte al punto $G$ en la solución del problema. De otra parte, $O G, G V$ y $G M$ se encuentran en el mismo plano $O N V$.

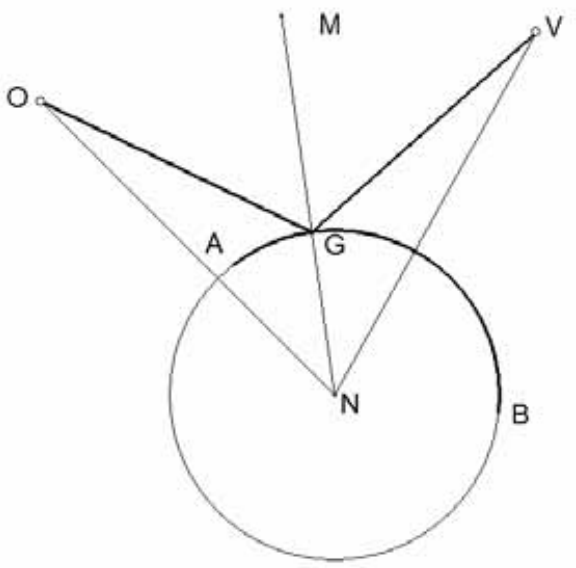

Figura 1. Problema de Alhacén para el caso en el que $O N \cong V N$

La solución del segundo caso, cuando objeto y observador se encuentran a distancias disímiles del centro del espejo, implica un camino muy tortuoso.

11 En estos puntos ocurre que las rectas $V A$ y $V B$ que no aparecen en la figura son tangentes a la circunferencia. 
De hecho, Alhacén presenta la solución después de ofrecer 6 lemas que atienden a construcciones auxiliares. Los lemas mencionados son las proposiciones 19 - 24 (Alhacén, V, 2.141 - 2.197). ${ }^{12}$ A continuación me ocuparé del método desarrollado por el filósofo árabe para resolver el problema asociado con los espejos esféricos convexos. Las construcciones auxiliares se presentan al final del apartado y se reenumeran atendiendo al orden en el que se van necesitando. La solución la presenta Alhacén en la proposición 25 (Alhacén, $\mathrm{V}, 2.198$ - 2.215).

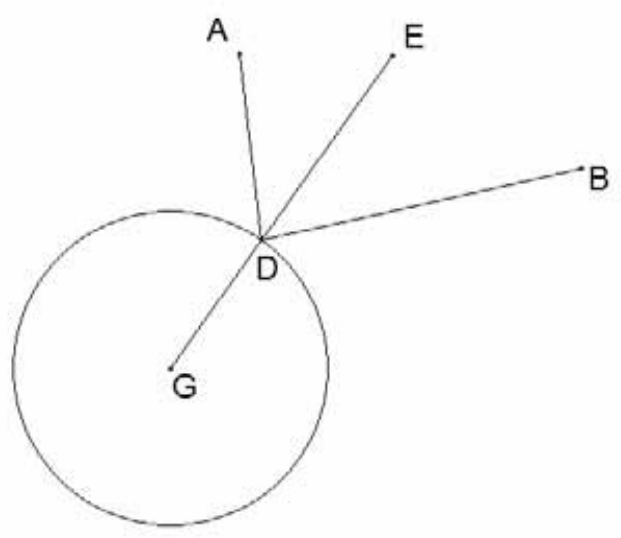

Figura 2. Formulación del Problema de Alhacén

Imaginemos un espejo esférico de centro $G$, un observador ubicado en $A$ y un objeto ubicado en $B$. La figura 2 muestra el corte de la esfera con el plano que contiene a los puntos $A, B$ y $G$. El punto de reflexión ha de encontrarse en el mismo plano, en consecuencia ha de hallarse sobre la circunferencia aludida. El problema se puede formular así: dada una circunferencia de centro $G$ y los puntos exteriores $B$ (objeto) y $A$ (observador), se pide hallar la ruta del rayo de luz que va desde $B$ hasta $A$ pasando por $D$ (un punto en el espejo cuya normal es $D E$ ) de tal manera que los ángulos $B D E$ y $A D E$ sean congruentes. A continuación (i) presento la solución de Alhacén; (ii) formulo algunas va-

12 Para un análisis y presentación de los lemas, véase SABRA, A. I., (1982), pp. 299-324; SMITH, M. (2008a), «Alhacen's Approach to «Alhazen's Problem»», Arabic Sciences and Philosophy, vol. 18 pp. 143-163. 
riaciones en la presentación de la construcción auxiliar requerida; (iii) presento las claves de la demostración; (iv) sugiero una hipótesis plausible que podría explicar el camino que condujo a Alhacén a concebir una solución tan compleja; (v) presento el lema 6; (vi) presento el lema 2; (vii) muestro la extensión del método general para ocuparse de los espejos convexos (cilíndricos y cónicos); y, por último, (viii) recojo la conclusión final.

(i) Presentación de la solución. Se construyen los segmentos auxiliares $F M$ y $F K$, uno a continuación del otro sobre la misma recta, de tal manera que $F M \cong B G$ y $F K \cong G A$ (Figura 3 ). ${ }^{13} O$ es el punto medio de $M K$ y $O C$ es una perpendicular a $M K$. El punto $C$ se elige de tal manera que el ángulo $O C K$ es la mitad del ángulo $B G A$. A continuación se halla un punto $S$ sobre $C O$ que satisface la siguiente condición: si desde ese punto se traza la recta $S F$, ésta va a cortar la recta $C K$ en un punto $P$ tal que $\frac{S P}{P K}=\frac{B G}{G D}$, siendo $G D$ el radio de la esfera. Este paso se lleva a cabo con la construcción auxiliar 1. Como veremos más adelante, la solución del problema depende esencialmente de la posibilidad de encontrar este punto $\mathrm{S}$.

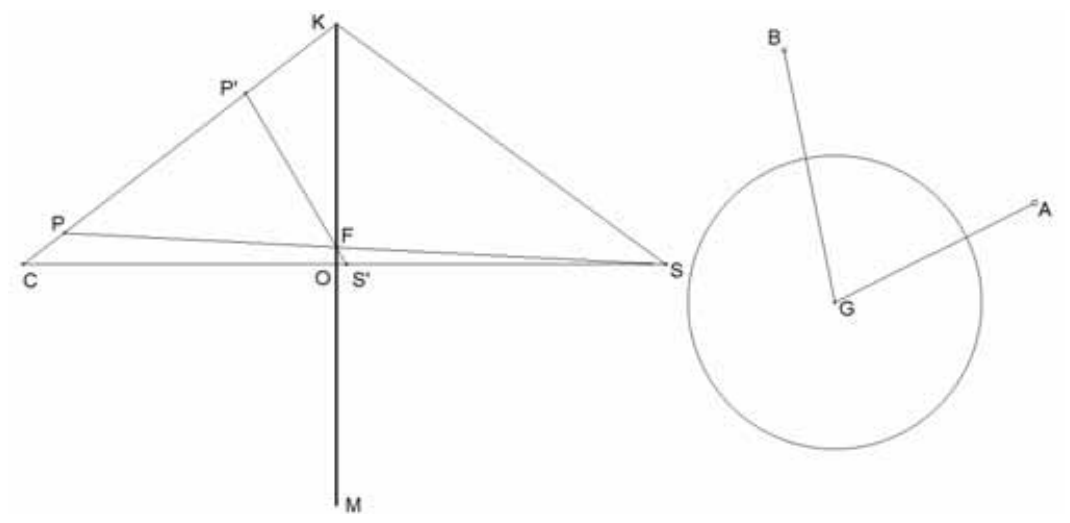

Figura 3. Problema de Alhacén (planteamiento y preámbulo)

13 Alhacén no exige la igualdad de los segmentos como aquí se sugiere, él pide que la construcción satisfaga la proporción $\frac{F M}{F K}=\frac{B G}{G A}$. Como la elección es arbitraria, podemos facilitar el razonamiento si la construcción auxiliar replica la medida de los segmentos $B G$ y GA (Alhacén, V, 2.200). 
No hay sólo un punto que satisface la condición impuesta en la construcción auxiliar. Hay dos puntos $S$ y $S^{\prime}$. Alhacén demuestra que si ninguno de los ángulos $C K S$ o $C K S$ ' es mayor que un ángulo recto, no habrá, entonces, solución para el problema. ${ }^{14}$ Imaginemos, pues, que $C K S$ es mayor que un ángulo recto. A continuación se construye el ángulo $B G E$ igual al ángulo $S P K$. La intersección de $G E$ con la circunferencia es el punto buscado $D$ (Figura 4). Probaremos, entonces, que $D$ satisface la condición exigida, a saber, los ángulos $B D E$ y $A D E$ han de ser congruentes (Sección (iii)).

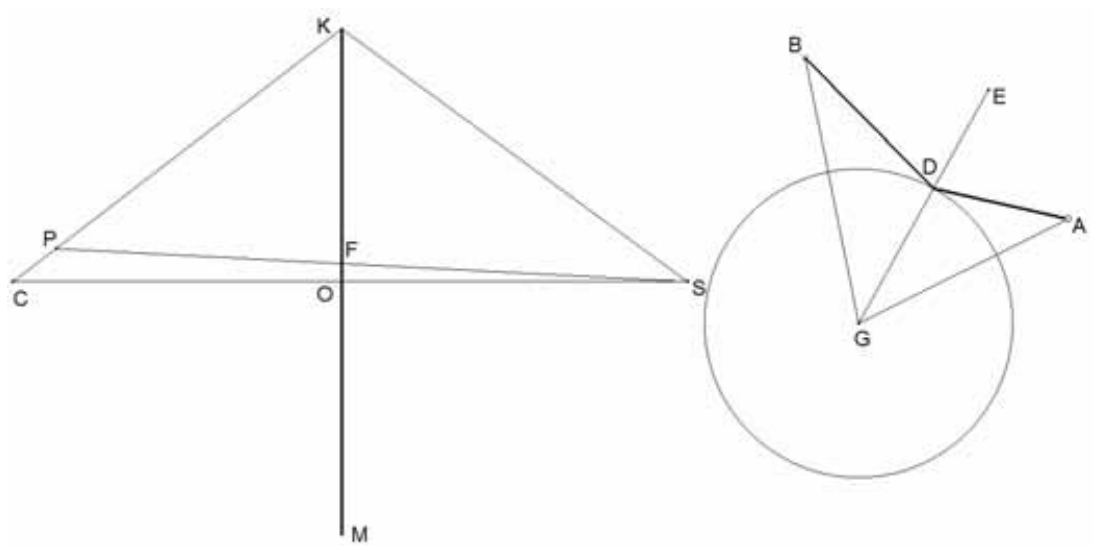

Figura 4. Problema de Alhacén (caso en el que $\angle C K S>90^{\circ}$ )

(ii) Variaciones en la presentación de la construcción auxiliar. La ubicación de la construcción al margen es completamente irrelevante. En consecuencia y a diferencia de Alhacén, haremos la construcción sobre la disposición inicial para facilitar la contemplación de las congruencias y semejanzas requeridas. Para ello, construimos el segmento $G K$, a continuación de $B G$, de tal manera que $A G \cong K G$. Para que el lector pueda seguir la variación propuesta, rebautizaremos los puntos $\mathrm{G}$ y B con las alusiones $G-F, B-M$. De esa manera se pueden seguir las descripciones en uno u otro esquema. Después replicamos todas las construcciones enunciadas en la presentación de la prueba. La figura 5 exhibe la nueva organización.

14 Este resultado se justificará más adelante. 


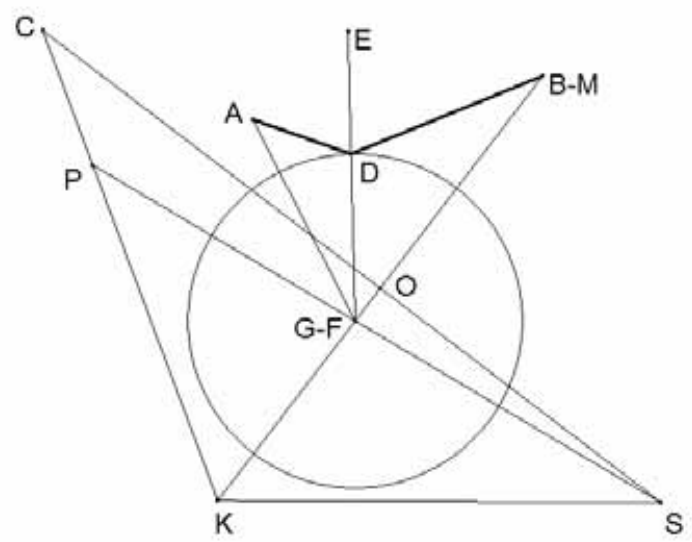

Figura 5. Problema de Alhacén (reubicación de la construcción auxiliar)

(iii) Demostración. Para efectos de la demostración se agregarán los siguientes elementos. La tangente a la circunferencia por $D$ que corta a $B G$ en el punto $N$; el punto $Q$ sobre $B G$ de tal manera que los ángulos $G D Q$ y $O K C$ sean congruentes; la perpendicular $B Z$ a $Q D$; el punto $I$ sobre $D Z$ de tal manera que $D Z \cong Z I ; D L$ paralela a $B I$, con $L$ sobre $B G$; el punto $H$ sobre $B G$, de tal manera que los ángulos $H D L$ y $B G A$ sean congruentes (Figura 6).

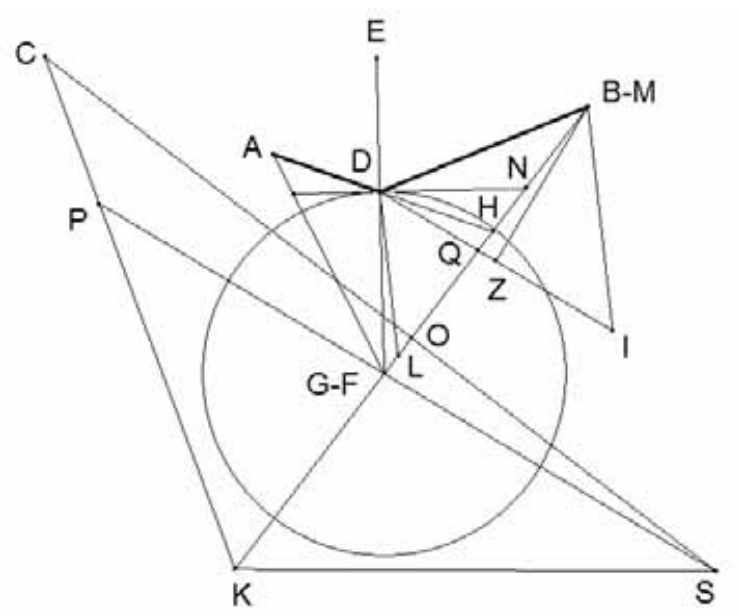

Figura 6. Problema de Alhacén (elementos de la prueba) 
Los elementos adicionales de la construcción conducen a establecer que $\angle Q D N=\frac{\angle H D L}{2}$, y $\angle B D Q=\frac{\angle B D L}{2} \cdot{ }^{15}$ Estos dos resultados contribuyen a mostrar que $\angle B D N \cong \angle N D H$. En consecuencia, sus respectivos ángulos complementarios $\angle B D E$ y $\angle H D G$ son congruentes. Ahora bien, si $H, D y$ $A$ fuesen colineales (Conjetura), de ello se podría concluir que $\angle B D E \cong \angle E D A$, que es lo que se busca.

Para probar la conjetura, prolongamos $H D$ (Figura 7) hasta cortar $G A$ en un punto $W$; por tanto $W$ es colineal con $H$ y $D$. Mostraremos que $W$ coincide con $A$. Sea el triángulo $G H W$, trazamos $H T$ paralelo a $B D$ (con $T$ sobre $G D)$.

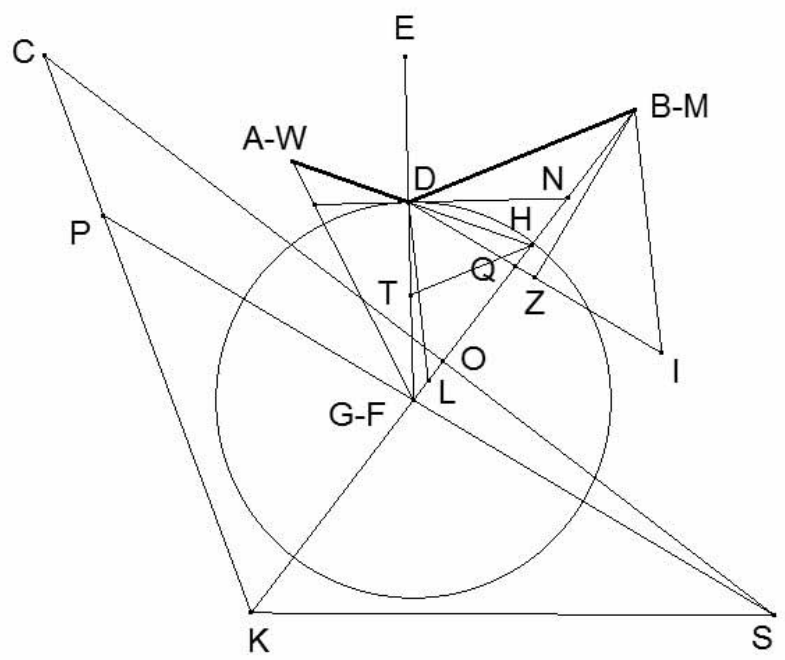

Figura 7. Problema de Alhacén (conjetura)

Dado que $\frac{S P}{P K}=\frac{B G}{G D}$ y $\angle B G D \cong \angle S P K$ (por construcción), se puede con-

15 En el primer caso, dado que $\angle Q D N+\angle G D Q$ y $\angle O C K+\angle O K C$ son rectos, y $\angle O K C \cong \angle G D Q$, se sigue $\angle Q D N \cong \angle O C K$. Además $\angle O C K=\frac{\angle B G A}{2}$ por construcción. En el segundo caso, $D L$ y $B I$ son paralelas, mientras que el triángulo $B D I$ es isósceles, pues $B Z$ es una perpendicular a $D I$ trazada en el punto medio $Z$. 
cluir en forma crucial que $S P K \approx \triangle B G D$. Las construcciones adelantadas también permiten establecer las siguientes semejanzas: $\triangle F P K \approx \triangle Q G D, \triangle B D Q \approx \triangle S K F, \triangle B Q Z \approx \triangle S O F .{ }^{16}$ A partir de estas últimas se puede inferir la proporción: $\frac{I Q}{Q D}=\frac{B G}{G A}$. De otro lado, la semejanza $\triangle L D Q \approx \triangle B Q I$ más el hecho de que $B D I$ es isósceles, imponen la proporción: $\frac{I Q}{Q D}=\frac{B D}{D L}$. A partir de estos resultados se infiere: (1) $\frac{B D}{D L}=\frac{B G}{G A}$.

En otra dirección, de la semejanza $\triangle H D L \approx \triangle H W G$ se infiere (2) $\frac{H D}{D L}=\frac{H G}{G W} \cdot{ }^{17}$ De la semejanza $\Delta G T H \approx \Delta G D B$, se establece $\frac{B D}{H T}=\frac{B G}{G H}$. Como $H D \cong H T$ (pues $\triangle T H D$ es isósceles, ya que $\angle B D E \cong \angle H D G$ (según los pasajes iníciales de la prueba) y $\angle B D E \cong \angle H T D$ ), se puede sustituir éste resultado en la proporción anterior y obtener: (3) $\frac{B D}{H D}=\frac{B G}{H G}$. Tomando $H D$ como media proporcional entre $B D$ y $D L$, se puede escribir: $\frac{B D}{D L}=\left(\frac{B D}{H D}\right)\left(\frac{H D}{D L}\right)$ para substituir (2) y (3) y concluir: (4) $\frac{B D}{D L}=\left(\frac{B G}{H G}\right)\left(\frac{H G}{G W}\right)$.

Sustituyendo (1) en (4) concluimos: $\frac{B G}{G A}=\left(\frac{B G}{H G}\right)\left(\frac{H G}{G W}\right)=\frac{B G}{G W}$, lo que nos permite defender que $W$ coincide con $A$ y, en consecuencia, $A, D$ y $H$ han de ser colineales (conjetura probada).

(iv) Hipótesis que exhibe una posible lógica del descubrimiento. ¿Cómo pudo Alhacén concebir un procedimiento tan complejo para dar con la solución del problema? Esta es una pregunta que quizá nunca logremos descifrar.

16 La primera se infiere del hecho de que los ángulos $F P K$ y $B G D$ son congruentes por construcción, así como lo son los ángulos $P K F$ y $Q D G$. La segunda se infiere porque (i) $S K F$ unido a $F K P$ resulta congruente con $B D Q$ unido con $Q D G$ (que es congruente a PKF) y (ii) $D B Q$ es congruente con $F S K$ por la semejanza previa establecida entre los triángulos $S P K$ y $B D F$. La tercera se infiere porque los dos triángulos son rectángulos y los ángulos $Q B Z$ y $O S F$ son congruentes.

$17 \angle H D L \cong \angle B G A$ por construcción y $H$ es colineal con $B$ y $G$. 
De un lado, los pasos preliminares recuerdan los métodos empleados por los griegos para hallar una media proporcional entre dos segmentos dados: (i) disponerlos uno a continuación del otro sobre una recta; (ii) hallar el punto medio de la composición; (iii) trazar una perpendicular por el punto de reunión de los dos segmentos; $y$, por último (iv), encontrar sobre dicha perpendicular un punto que satisface condiciones adecuadas para la solución (en este caso, el punto debe ser la intersección con la circunferencia que se centra en el punto medio y pasa por los dos extremos libres de los segmentos reunidos). ${ }^{18}$ La familiaridad de Alhacén con los métodos griegos pudo guiar los pasos iniciales. De otro lado, la solución del caso simple, aquel en el que objeto y observador equidistan del espejo, pudo ofrecer las pistas siguientes. ${ }^{19}$ Sumadas las dos ideas seminales, pudo ser que Alhacén, después de suponer que objeto y observador equidistan del centro del espejo, reuniera los segmentos $G B$ y $G A^{\prime}$ - $G K^{\prime}$ ' en la figura 8 - uno a continuación del otro sobre la misma recta. El punto $G$ define ya el punto medio que coincide con la intersección de los dos segmentos por donde se ha de trazar una perpendicular. A continuación, si ésta sugerencia es plausible, Alhacén pudo determinar la propiedad esencial del punto que habría de buscar sobre dicha perpendicular, a saber el punto $C$ '. $C^{\prime}$ es el corte de la recta $B A^{\prime}$ con la perpendicular a $B K^{\prime}$ por el punto $G$. Dado que los triángulos $A^{\prime} G B$ y $K^{\prime} C^{\prime} B$ son isósceles y semejantes, resulta que los ángulos $K^{\prime} C^{\prime} G$ y $B G D$ (la bisectriz de $A^{\prime} G B$ ) son congruentes. La Figura 8 muestra los rasgos sobresalientes de la solución de Alhacén para este caso particular. Utilizo las letras primadas para referirme a los mismos elementos de la solución general. En la Figura 8 el punto $C$ ' también se denomina $P$ '. Se puede esperar, dado que ya se sabe que la solución debe buscarse sobre la bisectriz de BGA, que la figura auxiliar replique un triangulo semejante a $B G D$. Como $G$ y $K^{\prime}$ ya forman parte de la construcción auxiliar y $\angle B G D \cong \angle G C^{\prime} K^{\prime}$, se puede encontrar un punto $S^{\prime}$ sobre $G C^{\prime}$ tal que $\angle B D G \cong \angle C^{\prime} K^{\prime} S^{\prime}$. De esa manera, los triángulos $B D G$ y $P^{\prime} K^{\prime} S^{\prime}$ resultan semejantes.

18 EuCLIDES, (1956), The thirteen books of the Elements, New York, Dover Publications, INC. Traducción al inglés y edición a cargo de Thomas L HEATH. VI, 13.

19 Mark Smith propone una estrategia que atiende también a esta segunda recomendación. Sin embargo, Smith no se concentra en la construcción auxiliar inicial, sino en los elementos agregados para ofrecer la prueba final. Esto hace que las dos propuestas, la de Smith y la mía, subrayen elementos diferentes aunque pudiesen estar emparentados. SMITH, A. M. (2006), xlix-li. Una síntesis del análisis también está presente en SMith, A. M. (2008a), pp. 146-151. 


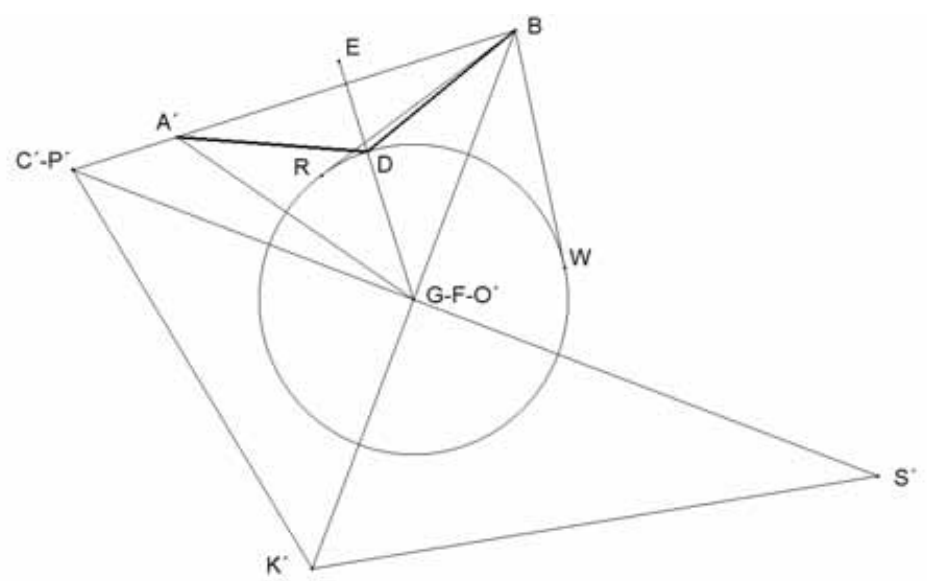

Figura 8. Solución al Problema de Alhacén en el caso en el que $G A^{\prime} \cong G B$

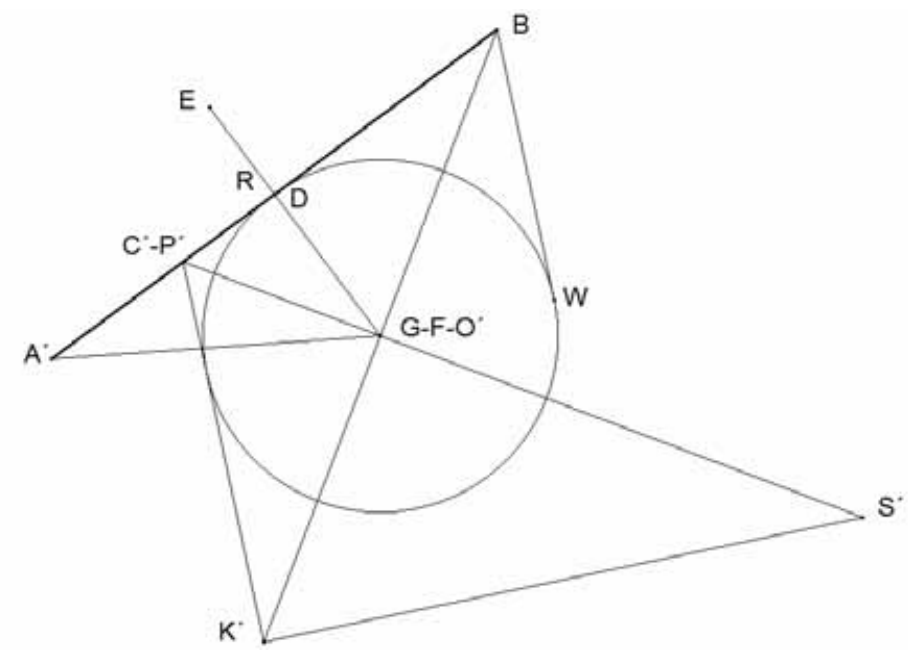

Figura 9. Solución al Problema de Alhacén en el caso en el que $A$ ' cae sobre la tangente $B R$.

$B R$ y $B W$ (tangentes a la circunferencia que define el espejo) determinan el sector en donde es factible encontrar una solución al problema. Cuando $A$ ' cae sobre la tangente $B R$ (como muestra la figura 9), estamos bordeando el límite para una solución posible. En este caso, $B D G$ es un ángulo recto y así mismo debe serlo $P^{\prime} K^{\prime} S^{\prime}$. Ello muestra por qué Alhacén advierte que para 
tener una solución al problema ha de ocurrir que el ángulo CKS (en la figura 7) sea mayor que un ángulo recto. ${ }^{20}$

Ahora bien, conocida la solución del caso elemental, Alhacén pudo evaluar situaciones emparentadas que condujesen a la misma solución, tratando de establecer las modificaciones y los invariantes básicos. Cualquier punto $A$ que se encuentre sobre la semirrecta $D A$ ' ha de conducir a la misma solución $D$, siempre que tanto $B$ como las características geométricas del espejo se conserven. Así entonces, el punto $D$ ya no se encuentra en la bisectriz de $A G B$. Cuando $A$ se desplaza desde $A$ ' hasta la nueva posición, la bisectriz rota de $G E$ a $G U$. La figura 10 muestra el caso. La solución al problema debe conducir al punto $D$. Los puntos $N$ y $H$, por razones obvias se mantienen invariantes ( $N$ es el corte de $B K^{\prime}$ con la tangente en $D ; H$ es el corte de $A^{\prime} D$ con $B K^{\prime}$ ). También resulta invariante el triangulo $B D G$. Imaginemos, pues, que Alhacén replica los pasos iniciales de la construcción auxiliar del caso simple. Pudo, entonces, disponer $G B$ y $G K(G K \cong G A)$ uno a continuación del otro para trazar después la perpendicular a este segmento por $G$. Si a continuación se construye el ángulo $G C^{\prime \prime} K\left(C^{\prime \prime}\right.$ sobre la perpendicular) de tal manera que $\angle G C^{\prime \prime} K \cong \angle A G U$ (la bisectriz de $A G B$ ), es claro que este ángulo ya no permite la determinación de $D$, que ahora no cae sobre dicha bisectriz. Como ya conocemos la solución, podemos construir los ángulos $G P K$ (con $P$ sobre $C^{\prime \prime} K$ ) y $P K S$ (con $S$ sobre $P G$ ) de tal manera que $\angle G P K \cong \angle B G D$ (mitad de $\left.A^{\prime} G B\right)$ y $P K S \cong G D B$. Así las cosas, el triángulo $S P K$ resulta semejante con el triángulo invariante $B D G$. Se puede probar, primero, que el punto $S$ cae precisamente sobre la perpendicular a $B K$ trazada en el punto medio $O$ y, segundo, que el ángulo $O S P$ es congruente con el ángulo $E G U$ (la rotación de las bisectrices como efecto del desplazamiento de $A) .{ }^{21}$ Dado que ahora el protagonismo se puede concentrar sobre la mediatriz $S O$, podemos prolongar $K C^{\prime \prime}$ hasta cortar a $S O$ en $C$.

(Fase de construcción). ${ }^{22}$ En ese orden de ideas, si nos dan inicialmente $B$ y $A$ (y desconocemos $A$ ), podemos trazar la perpendicular por el punto medio $O$, y después de trazar el ángulo $O C K$ congruente con la bisectriz de $B G A$, hemos de hallar $S$, sobre dicha perpendicular, de tal manera que podamos garantizar la colinealidad de $S, G$ y $P$, por un lado, y la semejanza de los

20 También se advierte que sólo hay solución si la recta $A^{\prime} B$ no corta a la circunferencia.

21 Estos resultados se pueden defender acudiendo a las construcciones que justificaron la demostración general.

22 Más adelante se entenderá el sentido de la introducción en paréntesis para el párrafo. 
triángulos $S P K$ y $B G D$ (aun cuando $D$ también se desconoce). Así las cosas, después de construir el triángulo rectángulo $C O K$, se busca sobre $C O$ el punto $S$ a partir del cual se puede trazar la recta $S G$ que corta a $C K$ en $P$ de tal manera que $\frac{S P}{P K}=\frac{B G}{G D}$. El hecho de que desconozcamos la posición precisa de $D$ no es óbice para adelantar la tarea, toda vez que la magnitud de $G D$, donde quiera que se encuentre $D$, coincide con el radio de la circunferencia. Esta construcción garantiza la colinealidad $S-G-P$ y da pie para garantizar la semejanza buscada si a continuación construimos $\angle B G E \cong \angle S P K$. Es, entonces, muy posible que Alhacén partiese de la solución del caso simple y, a continuación, procurase hallar los invariantes al modificar dicho caso. Más adelante veremos que este ejercicio metodológico se repite para situaciones más complejas (los espejos cilíndricos-convexos y los espejos cónicos-convexos).

De ser correcto el procedimiento de descubrimiento que hemos esbozado, hemos de preguntar, entonces, por la similitud o diferencias con los métodos de análisis y síntesis propuestos por Euclides en el Libro XIII de sus Elementos. En la traducción que sugiere Heath de esos difíciles pasajes, teniendo en cuenta las aclaraciones de Pappus, el análisis es el método mediante el cual aceptamos como hallado lo que estamos buscando, para después indagar por la causa antecedente de esto último y continuar así hasta llegar a algo ya conocido o perteneciente a los primeros principios. En el caso de la síntesis que le sigue, recorremos el camino inverso; esto es, partimos de lo ya conocido o de los primeros principios a los que arribamos con el análisis y, una vez garantizada la reversibilidad necesaria del proceso anterior, avanzamos hacia la construcción formulada como problema. ${ }^{23}$ Heath, siguiendo a Hankel, propone dos fases en el análisis: primero la transformación, en la que nos movemos desde la supuesta solución al problema hasta hallar una nueva construcción que no pertenece a los datos originales; y, segundo la resolución en la que se prueba que todas las partes restantes de la nueva construcción son conocidas. En formas análoga, la síntesis consta también de dos fases: la construcción en la que se sigue el proceso inverso de la resolución del análisis — garantizada la reversibilidad con carácter necesario-; y la demostración en la que se prueba que la construcción así obtenida satisface las condiciones exigidas inicialmente en el enunciado del problema.

Imaginemos, pues, que el procedimiento descrito exhibe efectivamente un camino de descubrimiento; y supongamos, también, que dicho procedimiento encarna la dupla análisis-síntesis. Veamos ahora si podemos identificar las

23 EuCLIDES, (1956), vol. 1, pp. 137-142. 
fases señaladas. Supongamos que la circunferencia y los puntos $A$ y $B$ (Figura 10) son dados y nos piden hallar el punto que satisface las condiciones del punto de reflexión. Imaginemos que $D$ sea ese punto.

Fase de transformación. Trazamos la bisectriz $G U$ al ángulo $A G B$. Trazamos la semirrecta $D A$ y hallamos $A^{\prime}$ sobre ella, de tal manera que $G A^{\prime} \cong G B$. $D$ es también la solución al problema de Alhacén si hubiesen sido dados $A^{\prime}$ y $B$ originariamente. Trazamos $B A^{\prime}$ y obtenemos el corte $C^{\prime}$ con la perpendicular a $G B$ por $G$ (Figura 8). Se construye el triángulo isósceles $\mathrm{BC}^{\prime} \mathrm{K}^{\prime}$ y el triángulo $C^{\prime} K^{\prime} S^{\prime}\left(A^{\prime} G \cong K^{\prime} G\right)$ forzando la semejanza con el triángulo $B D G$.

Fase de resolución. Imaginamos ahora que un punto virtual se desplaza a lo largo de la semirrecta $D A$, desde $A^{\prime}$ hasta llegar nuevamente a $A$. En esa transformación exigimos, primero, la invarianza de la semejanza entre los triángulos $B D G$ y $S P K$ (con $S$ y $C$ sobre la mediatriz de $B K$ (Figura 10), $P$ sobre la recta $C K, G K$ de idéntica longitud a la que existe entre $G$ y el punto virtual y el ángulo $S C K$ congruente con la bisectriz al ángulo formado entre $B, G$ y el punto virtual), y segundo, que la rotación de la bisectriz $(E G U)$ se replique en el ángulo $O S G$.

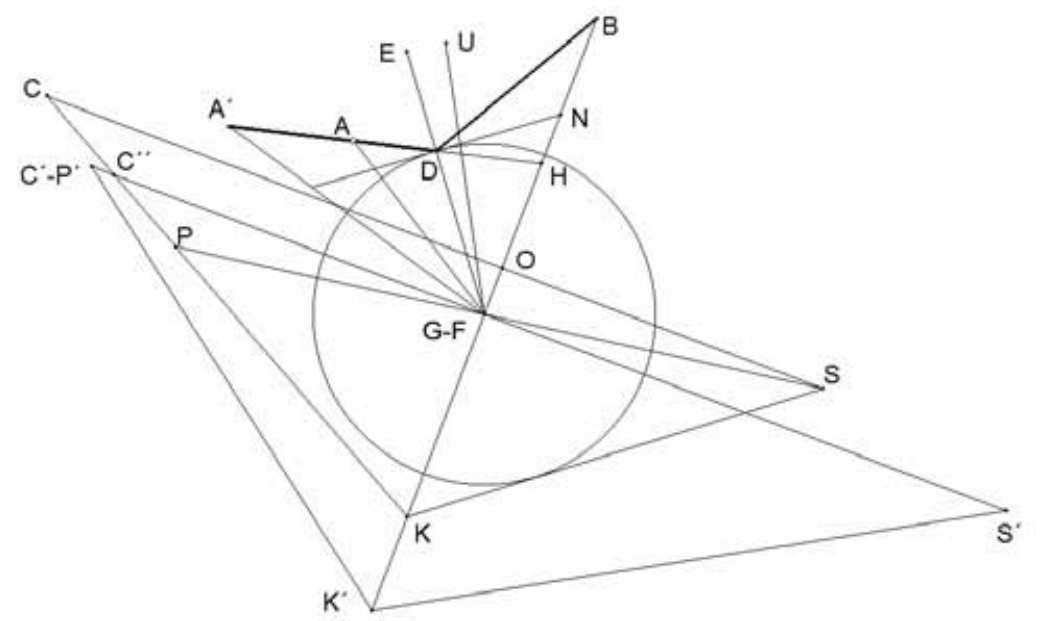

Figura 10. Solución al Problema de Alhacén en el caso en el que $A$ cae en la recta $D A$ '.

Fase construcción. Es la fase descrita en el párrafo que inicia con la anotación (Fase construcción).

Fase de demostración. Es la fase descrita con el numeral (iii). 
(v) Construcción auxiliar 1 (Lema 6). Nos ocuparemos ahora de las dos construcciones auxiliares (Lemas 6 y 2) que hacen posible la solución general para el caso de los espejos esféricos convexos. Dado el triángulo rectángulo $A B G$ ( $A B G$ recto) y un punto $D$ sobre uno de los catetos (para el caso, $B G$ ); dados también los segmentos libres $E$ y $Z$, se pide hallar la recta que pasa por $D$ y corta a la hipotenusa $A G$ en $Q$ y al otro cateto $A B$ en $T$, de tal manera que $\frac{T Q}{Q G}=\frac{E}{Z} \cdot 24$

La solución exige adelantar las siguientes construcciones (Figura 11). Trazar $A D$. Trazar $D M$ paralela a $B A, M$ en el corte con $G A$. Construir la circunferencia que pasa por $G, D$ y $M$. Construir $\angle D M C \cong \angle G A D$ con $C$ sobre la circunferencia. Construir el segmento $H$ de tal manera que $\frac{A D}{H}=\frac{E}{Z}$. A partir de $C$ se halla la recta que corta a $G M$ en $L$ y a la circunferencia en $N$ de tal manera que $L N \cong H$. Este procedimiento exige una nueva construcción auxiliar (Construcción Auxiliar 2). Ahora se traza la recta $N D$ y se obtienen los cortes $Q$ y $T$ con $A G$ y $A B$. La recta $N D$ es la recta buscada.
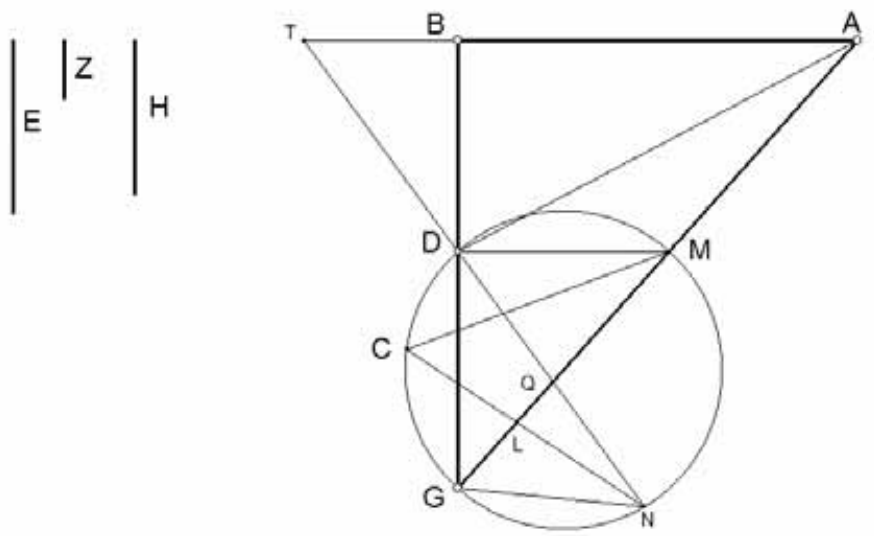

Figura 11. Lema 6

24 Alhacén, V, $2.193-2.197$ 
Dado que $\triangle N Q L \approx \triangle D Q A$ se tiene $\frac{A Q}{Q N}=\frac{A D}{N L} \cdot{ }^{25}$ De la semejanza $\Delta T Q A \approx \Delta N Q G$ se concluye: $\frac{T Q}{Q G}=\frac{A D}{N L}$. Como el lema 2 garantiza la construcción de $N L \cong H, y \frac{A D}{H}=\frac{E}{Z}$ (por construcción) se puede concluir, entonces, $\frac{T Q}{Q G}=\frac{E}{Z}$, que era lo que se pretendía. ${ }^{26}$ La solución completa del Problema de Alhacén depende ahora de nuestra habilidad para trazar $C N$ de tal manera que la longitud de $L N$ coincida con la longitud de un segmento dado.

(vi) Construcción auxiliar 2 (Lema 2). Dada una circunferencia GAB de diámetro $G B$ y un segmento de recta $H Z$, se pide trazar una recta desde $A$ que corte la circunferencia adicionalmente en $D$ y al diámetro $G B$ en $E$, de tal manera que $E D \cong H Z$ (Alhacén, $\mathrm{V}, 2.158-2.166$ ). ${ }^{27}$

La solución del problema exige las siguientes construcciones (Figura 12). Trazar $A B$ y $A G$. Se dispone $H Z$ sobre la recta $G B$. Se traza $H M$ paralelo a $G A$ y el ángulo $L H Z$ congruente con el ángulo $A B G .{ }^{28}$ Se proyecta ortogonalmente $Z$ sobre $H M$ (en $T$ ) y sobre $H L$ (en $N$ ). Se construye la cónica (hipérbola) que pasa por $T$ y tiene como asíntotas a $Z N$ y $H L .{ }^{29}$ Se traza la circunferencia de centro $T$ y radio $B G$ (puede ocurrir que corte la otra rama en dos puntos, en un punto o en ninguno). Sean $C$ y $C^{\prime}$ los cortes de dicha circunferencia con la

25 La semejanza se infiere de que $\angle N Q L \cong \angle D Q A$ (opuestos por el vértice) y $\angle D M C \cong \angle D N C$ ya que ellos subtienden el mismo arco $C D$ (EuCLIDES, (1956), III, 21) y $\angle D M C \cong \angle D A G$ (por construcción).

26 El lema 2 prevé dos soluciones, de donde se puede esperar también dos soluciones para el problema propuesto.

27 Los griegos se referían a esta clase de problemas con el término neusis.

$28 \quad M$ y $L$ se definirán con más precisión a continuación. Por lo pronto no requieren de una definición más precisa.

29 Alhacén sugiere usar el método de Apolonio (Cfr. Apolonio, (2000). Conics. Santa Fe, Green Lion Press. Edición preparada por Dana Densmore, II, 4). Pappus denominaba problemas sólidos a aquellos cuya solución requiere en forma necesaria el uso de superficies cónicas (cfr. KNORR, W. R., (1986), p. 341). La referencia necesaria a una cónica puede tomarse como indicio de la posibilidad de que el problema no se pueda resolver con el uso exclusivo de regla y compás. Para una demostración en esta dirección véase NEUMANN, P., (1998), «Reflections on reflection in a spherical mirror», en American Mathematical Monthly, 105, pp. 523-528. 
otra rama de la hipérbola en caso de que existan los cortes o coincidan en uno solo (si no hay corte, no habrá solución). Se traza $T C$ y se hallan los cortes $F, Q$ con las asíntotas $H L$ y $Z N$. Se traza la paralela a $T C$ por $Z$ y se definen los cortes $M, L$ con $H T$ y $H N$. Se construye el ángulo $B G D$ ( $D$ sobre la circunferencia) de tal manera que $\angle B G D \cong \angle H L Z$ y se traza $A D$ que corta a $B G$ en $E$. En este caso $E D \cong H Z$, que es lo que se quería. Si en lugar de tomar $C$ se hubiese tomado $C^{\prime}$ y se sigue el mismo procedimiento, el resultado conduce a otro punto $D^{\prime}$ que satisface también la condición impuesta.

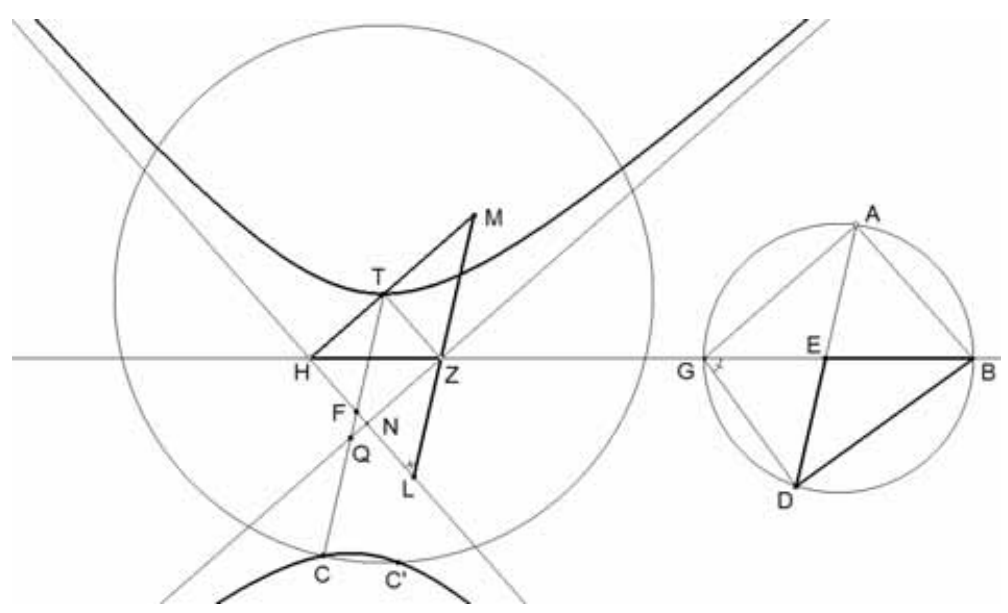

Figura 12. Lema 2

Dadas las semejanzas $\triangle H M L \approx \triangle G D B$ y $\triangle H M Z \approx \triangle D E B$, se tiene $\frac{B G}{B D}=\frac{L M}{M H}$ y $\frac{B D}{D E}=\frac{M H}{H Z}$, de donde concluimos (1) $\frac{B G}{D E}=\frac{L M}{H Z}$. De otro lado, dado que $Q C \cong T F$ (Apolonio II, 16) ${ }^{30}$ y $T F \cong L Z$ concluimos $Q C \cong L Z ; \mathrm{y}$ dado que $T Q \cong Z M$ inferimos (2) $L M=T Q+Q C=T C$. A partir de (1) y (2) tenemos $\frac{B G}{D E}=\frac{T C}{H Z}$. Finalmente, dado que $B G \cong T C$ por construcción, concluimos $D E \cong H Z$ que es lo que esperábamos construir.

30 Apolonio demuestra en II, 16 que si $Q$ y $Q^{\prime}$ son puntos arbitrarios sobre ramas diferentes de una hipérbola y $K$ y $K^{\prime}$ los cortes de $Q Q^{\prime}$ con las asíntotas, ocurre que $Q K \cong Q^{\prime} K^{\prime}$. 
(vii) Espejos cilíndricos y cónicos convexos. Las soluciones de Alhacén, si bien siguen el mismo esquema de razonamiento, se vuelven absolutamente complejas por lo extensas y lo escabroso de los giros en los argumentos. No obstante, la estructura profunda del razonamiento es simple, elegante y poderosa. Expongo en líneas generales el esquema de razonamiento y procuro resaltar el parecido de familia con el esquema propuesto en la lógica del descubrimiento. Alhacén concibe dos casos simples y un caso complejo. El caso complejo se resuelve atendiendo la solución de un caso simple para después hacer modificaciones que resaltan elementos invariantes. En primer lugar, observador y objeto pueden encontrarse en un plano que contiene al eje del cilindro o del cono. En este caso el punto de reflexión ha de encontrarse en la intersección de dicho plano y la superficie del espejo (a saber, una recta). El problema entonces se reduce a hallar la solución para el caso de un espejo plano. En el segundo caso, observador y objeto pueden encontrarse en un plano paralelo a la base del cilindro o del cono. El punto de reflexión ha de encontrarse, pues, en la intersección de dicho plano y la superficie del espejo, a saber una circunferencia. Este caso remite a hallar el punto de reflexión en un espejo esférico convexo. La Figura 13 exhibe los casos 1 y 2 para espejos convexos cilíndricos y cónicos. $A$ representa un objeto (o un observador), $B$ un observador (o un objeto) que se encuentra junto con $A$ en un plano que corta longitudinalmente bien sea al cilindro o al cono; $B$ ' un observador (o un objeto) que se encuentra con $A$ en un plano paralelo a la base del cilindro o del cono; $G$ y $G$ ' son los respectivos puntos de reflexión.
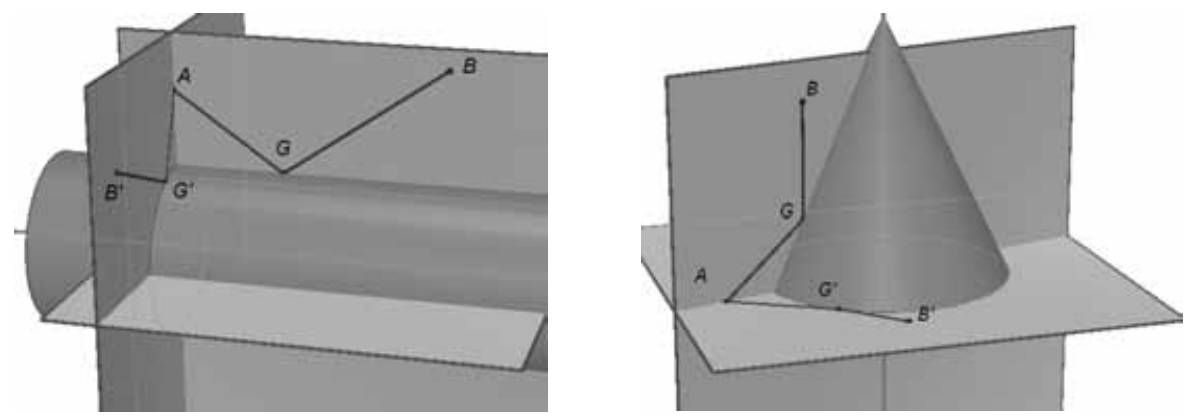

Figura 13. Problema de Alhacén, espejos cilíndricos o cónicos convexos (casos 1 y 2)

En el tercer caso - este es el caso más complejo e interesante-, no hay un plano que contenga al objeto, al observador y al mismo tiempo o bien sea 
paralelo a la base, o bien contenga al eje del cilindro o del cono. El esquema propuesto por Alhacén es el siguiente. Sea $A$ el observador y $B$ el objeto. Se determina un plano paralelo a la base (del cilindro o del cono) que contenga al punto $A$. El corte de este plano con la superficie del espejo es una circunferencia. Llamemos a esta circunferencia $S$. Después, sobre dicho plano se proyecta al punto $B$ en forma perspectiva desde el vértice del cono, o en forma ortogonal si se trata de un cilindro. ${ }^{31}$ Sea $G$ el vértice y $B$ ' la imagen de $B$ sobre el plano que contiene a $A{ }^{32} A$ continuación se resuelve el problema de Alhacén para los puntos $A$ y $B^{\prime}$. Este caso coincide con el caso de los espejos esféricos convexos. Sea $C^{\prime}$ la solución de dicho problema. $C^{\prime}$ se encuentra sobre $S$. A continuación se traza C'E' normal al cilindro o al cono. Luego se traza la recta que une a $C^{\prime}$ con $G$. Ahora se concibe el plano que contiene a $C^{\prime}$ y al eje del cono (o del cilindro). Es claro que $C^{\prime} G$ pertenece a dicho plano. Ahora se busca la intersección de dicho plano con la recta $A B$. Sea $K$ tal intersección. Finalmente se traza una perpendicular a $C^{\prime} G$ que pasa por $K$. La base de tal intersección $F$ se encuentra sobre
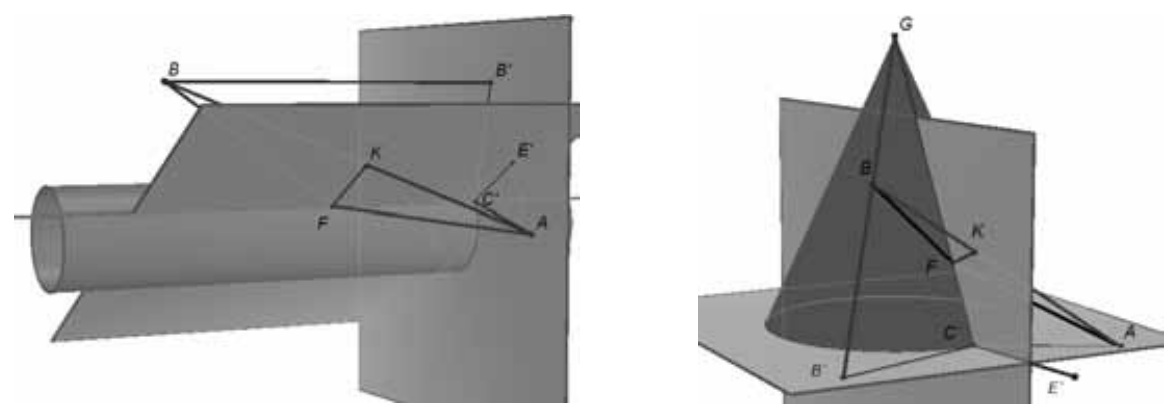

Figura 14. Problema de Alhacén, espejos cilíndricos convexos y cónicos convexos.

la superficie del espejo y determina el punto buscado. $F K$ es la normal a la superficie del espejo en el punto de reflexión. Es claro que $A F$ (rayo reflejado), $F K$

31 Se traza una recta desde el vértice que contenga al punto $B$. A continuación se halla la intersección de dicha recta con el plano paralelo a la base y que contiene a $A$. Si el espejo es cilíndrico bien podríamos pensar que se trata de un cono cuyo vértice se encuentra corrido al infinito. En ese orden de ideas, la proyección se vuelve ortogonal. (Este no es el lenguaje de Alhacén, por razones obvias).

32 Si se trata del cilindro, el vértice $G$ se concibe como un punto al infinito. 
(normal) y $B F$ (rayo incidente) se encuentran en el mismo plano $A B F \cdot{ }^{33}$ Alhacén procura demostrar que la condición de congruencia entre los ángulos $B^{\prime} C^{\prime} E^{\prime} \mathrm{y}$ $E^{\prime} C^{\prime} A$ no se pierde en la proyección. ${ }^{34}$ Por lo tanto los ángulos $A F K$ y $B F K$ son congruentes. La Figura 14 muestra los elementos descritos para los dos casos.

(viii) Conclusión. La exposición que Alhacén ofrece de la solución al problema que lleva su nombre oculta la lógica del descubrimiento. Nos encontramos con un ejercicio complejo, por la cantidad de movimientos abstrusos que supone, y brillante, por la contundencia del resultado. Hemos sugerido un procedimiento que podría develar aspectos centrales de la lógica del descubrimiento. Suponemos que se usan, primero, métodos emparentados con los procesos usados por los griegos para hallar medias proporcionales, $\mathrm{y}$, segundo, un mecanismo heurístico sencillo que se puede resumir así: (i) hallar la solución a un caso trivial, y (ii) deformar el caso trivial conservando los invariantes básicos hasta dar con las condiciones del caso complejo. Es probable que esta combinación encarne la reunión juiciosa de análisis y síntesis. De cualquier manera, la solución del problema para el caso de los espejos cónicos y cilíndricos exhibe nuevamente el patrón que hemos querido resaltar: solución de un caso trivial, seguido de una transformación que atiende los invariantes hasta dar cuenta de las condiciones complejas iniciales.

Recibido: 14 de junio de 2010

Aceptado: 4 de abril de 2011

$33 K$ está en el plano $A B F$, pues $K$ está en la recta $A B$.

34 La demostración no reviste mayor complejidad. 